\title{
In vitro quality of platelets during prolonged storage after washing with three platelet additive solutions
}

\author{
Nahreen Tynngård, M. Trinks and Gösta Berlin
}

\section{Linköping University Post Print}

N.B.: When citing this work, cite the original article.

This is the authors' version of the following article:

Nahreen Tynngård, M. Trinks and Gösta Berlin, In vitro quality of platelets during prolonged storage after washing with three platelet additive solutions, 2012, Vox Sanguinis, (102), 1, 3239.

which has been published in final form at:

http://dx.doi.org/10.1111/j.1423-0410.2011.01500.x

Copyright: Wiley-Blackwell

http://eu.wiley.com/WileyCDA/Brand/id-35.html

Postprint available at: Linköping University Electronic Press

http://urn.kb.se/resolve?urn=urn:nbn:se:liu:diva-74119 
In vitro quality of platelets during prolonged storage after washing with three platelet additive solutions

Nahreen Tynngård ${ }^{1,2}$, Marie Trinks ${ }^{1,2}$, and Gösta Berlin ${ }^{1,2}$

${ }^{1}$ Department of Clinical Immunology \& Transfusion Medicine, University Hospital,

Linköping, Sweden

${ }^{2}$ Department of Clinical and Experimental Medicine, Division of Transfusion Medicine, Linköping University, Linköping, Sweden

Correspondence to: $\quad$ Gösta Berlin, $\mathrm{MD}, \mathrm{PhD}$

Department of Clinical Immunology \& Transfusion Medicine University Hospital

SE-581 85 Linköping

Sweden

Telephone +46101033275 Fax +46101033282

e-mail: gosta.berlin@lio.se

Reprints will not be available from the author.

Running head: Washing of platelet concentrates with three PASs 


\begin{abstract}
Background and Objectives Patients with anaphylactic transfusion reactions require washed platelet concentrates (PCs) for subsequent platelet (PLT) transfusions. New PLT additive solutions (PASs) contain substances that might be beneficial for preservation of PLT function during storage. This study compares the quality of PLTs washed and stored with T-Sol, Composol or SSP+.
\end{abstract}

Study Design and Methods 15 buffy-coats were pooled and divided into three parts. PCs with 30\% plasma and 70\% PAS (T-Sol, Composol or SSP+) were prepared. Washing was performed on day 5 of storage. Ten PCs were prepared and washed with each PAS. In vitro variables including haemostatic function (clotting time and clot retraction) were analysed on day 5 before, directly after and up to 2 days after washing.

Results Swirling was well preserved and $\mathrm{pH}$ within acceptable limits (6.4-7.4) during storage for all PASs. The PLT number was reduced by washing for all PASs and T-Sol PCs had a further decrease during storage. PLTs in T-Sol were spontaneously more activated and had lower capacity to respond to an agonist than Composol or SSP+ PLTs. The haemostatic function was only slightly changed by washing and during post washing storage.

Conclusion PLTs washed with T-Sol, Composol or SSP+ had good in vitro quality for two days after washing despite absence of glucose. PLTs in T-Sol were more affected by the washing procedure and subsequent storage than Composol or SSP+ PLTs as judged by higher spontaneous activation.

Keywords: Platelet concentrate, platelet additive solutions, platelet function, platelet washing, platelet storage 


\section{Introduction}

Platelets (PLTs) are stored in plasma or in a combination of plasma and a PLT additive solution (PAS). Plasma constituents can cause anaphylaxis and febrile non-haemolytic transfusion reactions [1]. A patient who develops an anaphylactic transfusion reaction needs washed PLT concentrates (PCs) without plasma for subsequent transfusions. PCs should be transfused within a few hours after washing according to guidelines [2] but it would be logistically beneficial to be able to store washed PCs for a longer period before transfusion. Composol (PAS-D) and SSP+ (PAS-E) are new PASs containing substances that have been suggested to be advantageous for preservation of PLT function $[3,4]$ and thus might allow washed PCs to be stored for more than a few hours after washing. There are only a few studies showing in vitro quality of PCs washed and stored with these PASs [5, 6] and there is a lack of studies analysing haemostatic properties.

We compared the in vitro quality of buffy-coat-derived PCs washed and stored with T-Sol (PAS-B), Composol or SSP+. The quality was assessed up to 2 days after washing by in vitro assays including assessment of haemostatic properties using free oscillation rheometry (FOR) [7]. 


\section{Materials and methods}

\section{PLT collection, preparation and storage}

Blood was drawn from healthy donors into "top-and-bottom" containers (Fenwal, Lake Zurich, IL, USA). Buffy-coats (BCs) were prepared as previously described [8]. The BCs were held at $22^{\circ} \mathrm{C}$ overnight without agitation. Fifteen $\mathrm{ABO}$-identical BCs were pooled and then divided into three equal parts. The OrbiSac system (Caridian BCT, Lakewood, CO, USA) was used for automatic processing of PCs from the BCs [9]. The first BC part was pooled with $300 \mathrm{ml}$ T-Sol (Fenwal), the second with Composol (Fresenius Kabi AG, Bad Homburg, Germany) and the third with SSP+ (MacoPharma, Mouvaux, France). Ten PCs with each PAS were prepared. The PCs were left to rest for one hour at $22^{\circ} \mathrm{C}$ and then transferred for storage on an agitator (Model LPR-3, Melco Engineering, Glendale, CA, USA) in an incubator (Helmer PC2200, Helmer, Noblesville, IN, USA) with a stable temperature of $22 \pm 2{ }^{\circ} \mathrm{C}$.

\section{Platelet washing}

On day 5 of storage the PCs were washed with T-Sol, Composol or SSP+, respectively, according to routine procedures at the Department of Clinical Immunology \& Transfusion Medicine, Linköping University Hospital, Sweden [8]. In short, a bag with $300 \mathrm{ml}$ of the PAS was connected to the PC with a sterile connecting device and the PAS was transferred to the PC. The PAS bag was removed and a transfer bag (Terumo Corp., Tokyo, Japan) was connected to the PC. The PC was centrifuged at $2790 \mathrm{~g}$ for $6.5 \mathrm{~min}$ at $22^{\circ} \mathrm{C}$. The plasma/PAS was transferred to the transfer bag. The procedure was repeated once. Finally, $300 \mathrm{ml}$ of the PAS was transferred to the PC. After washing, the PC was left to rest on the bench for one hour at $22^{\circ} \mathrm{C}$ and then on the agitator (Model LPR-3, Melco Engineering) in the incubator 
(Helmer PC2200) for one hour or more depending on the result of visual inspection of microaggregates prior to any sampling. The PCs were stored on the agitator for 2 days.

\section{Sampling}

On each sampling occasion $10 \mathrm{ml}$ was taken aseptically from the PC using a sampling bag (MacoPharma) and was used for various in vitro tests. Sampling was performed on day 5 pre wash, on day 5 post wash (6-60 min after washing), 1 day post wash (20-21 hours after washing) and 2 days post wash (43-45 hours after washing) for analyses of blood gases, metabolic variables, PLT concentration, mean PLT volume (MPV), PLT surface markers by flow cytometry, and FOR.

\section{Swirling}

Swirling was examined by visual inspection of the PCs and graded as $0,+,++$ or $+++(0=$ no swirling, $+++=$ maximum swirling).

\section{PLT concentration and MPV}

PLT concentration and MPV were measured with the haematology analysers, Micros 60 (ABX Diagnostics, Montpellier, France) and Cell-Sapphire (Abbott Diagnostics Division, Abbot Park, IL, USA), respectively.

\section{Blood gases and metabolic variables}

$\mathrm{pH}, \mathrm{pO}_{2}, \mathrm{pCO}_{2}$ were measured at $37^{\circ} \mathrm{C}$ with a blood gas analyser ( $\mathrm{ABL} 725$, Radiometer Medical ApS, Copenhagen, Denmark) immediately after sampling. Glucose, lactate and albumin concentrations were measured in autologous plasma with a chemistry analyser (Advia 1650, Bayer Healthcare LLC Diagnostics Division, Tarrytown, NY, USA). 


\section{PLT surface markers}

PLT surface markers P-selectin (CD62P) and GPIb (CD42b) were analysed by flow cytometry.

For measurement of spontaneous P-selectin expression and GPIb anti-CD62P-FITC (polyclonal chicken anti-human CD62P, Diapensia HB, Linköping, Sweden) and anti-CD42bPE (monoclonal mouse anti-human CD42b, DakoCytomation, Glostrup, Denmark), respectively, were used. As negative controls, IgY-FITC (non immune chicken IgY, Diapensia HB) and anti-IgG2a-PE (monoclonal mouse anti-human IgG2a, DakoCytomation) were used. After addition of PLTs to the antibodies, the sample was incubated for $20 \mathrm{~min}$ before the reaction was stopped by addition of HEPES buffer.

For measurement of activator induced P-selectin expression PLTs were added to anti-CD62PFITC and after incubation for $10 \mathrm{~min}$, a thrombin-receptor 1-activating peptide (TRAP-6, SFLLRN, Biotechnology Centre of Oslo, Oslo, Norway) was added (final concentration 0.06 $\mathrm{mmol} / \mathrm{l})$. After further incubation for $10 \mathrm{~min}$ the reaction was stopped by addition of HEPES. Flow cytometry was performed using the instrument Epics XL-MCL (Beckman Coulter Inc., Fullerton, CA, USA) as previously described [10].

\section{Clotting time and coagulum elasticity ( $\left.G^{\prime}\right)$}

Clot formation and clot retraction were studied by FOR with a rheometer (ReoRox 4, Medirox AB, Nyköping, Sweden). When analysing clotting time and clot retraction (clot elasticity, clot strength) the sample is added to a reaction chamber consisting of a sample cup with a cylinder (bob) attached to a shaft in the centre of the cup. When the reaction chamber is placed in the 
rheometer, the sample cup starts to oscillate. The change in the frequency and damping of the oscillation during clot formation and clot retraction is registered optically over time [7].

The PLT concentration was adjusted to $100 \times 10^{9}$ PLTs/l by dilution with plasma from an AB $\mathrm{D}+$ donor. The plasma was kept at $-70^{\circ} \mathrm{C}$ until use and thawed in a water bath $\left(37^{\circ} \mathrm{C}\right)$. The diluted PC sample was recalcified, activated with TRAP-6 and analysed in the ReoRox 4 [8].

Clotting time, maximum elasticity (G'max; final clot strength) and time between clotting time and G'max (time to G'max) were determined as previously described [8]. The mean change in elasticity $\left(\mathrm{G}^{\prime}\right)$ per min showing the speed of clot elasticity development (i.e. G'max/time to G'max) was calculated. An elasticity curve is shown in Fig. 1.

\section{Statistical analysis}

The results are presented as mean \pm standard deviation (SD). Three-way analysis of variance (ANOVA) with replication (repeated measures) was used to determine if there were any significant differences between before and after washing on day 5, between storage days after washing, between the PAS groups, and if washing- and/or storage-induced changes differed significantly between the PAS groups. If a significant difference was found before and after washing on day 5 or between storage days after washing, one-to-one paired two-tailed t tests were applied for comparisons between the test occasions within each PAS group. If a significant difference was found between the PAS groups, one-to-one paired two-tailed t tests were applied for comparisons between the PAS groups. A $P$-value of $<0.05$ was considered significant. Microsoft Office Excel 2003 (Microsoft Corp., Redmond, Washington, USA) was used for calculation of mean \pm SD and t tests. SPSS was used for ANOVA calculations (SPSS Inc., Chicago, IL, USA). 


\section{Results}

\section{Swirling}

Swirling was well maintained (+++) in all PCs on all storage days before and after washing.

\section{PLT number and MPV}

The number of PLTs in the PCs prior to washing was similar for the different PASs. Washing resulted in a loss of PLTs $(\boldsymbol{P}<0.05)$ by $16 \pm 7 \%$ for T-Sol PCs (mean \pm SD) , $22 \pm 6 \%$ for Composol PCs and $17 \pm 4 \%$ for SSP+ PCs. The number of PLTs per unit decreased to a similar level $(P>0.05)$ for all PASs $\left(305 \pm 35 \times 10^{9}\right.$ for T-Sol PCs, $286 \pm 37 \times 10^{9}$ for Composol PCs and $315 \pm 23 \times 10^{9}$ for SSP+ PCs; Fig. 2) which was confirmed by ANOVA $(P>0.05)$. During storage after washing the number of PLTs did not change significantly in PCs with Composol or SSP+ whereas T-Sol PCs had a small but significant decrease in PLT number.

PLTs in the different PASs had similar MPV before washing (Fig. 1). Washing or subsequent storage in Composol did not affect MPV. Washing with T-Sol or SSP+ reduced MPV $(P<0.05)$. Subsequent storage increased MPV for PLTs in T-Sol or in SSP+ and 2 days post washing PLTs in T-Sol had higher MPV than in Composol or in SSP+. ANOVA confirmed significant differences in the washing and storage-induced changes of MPV between PASs.

\section{Blood gases and metabolic variables}

$\mathrm{pH}, \mathrm{pO}_{2}$ and $\mathrm{pCO}_{2}$ values are shown in Table 1. $\mathrm{pH}$ was within acceptable range (6.4-7.4; [11]) for all PCs on all storage days. $\mathrm{pH}$ for SSP+ PCs was not affected by washing but was reduced during subsequent storage $(P<0.05)$. Washing resulted in a $\mathrm{pH}$ reduction for T-Sol 
and Composol PCs. ANOVA confirmed significant differences in the washing- and storageinduced $\mathrm{pH}$ change between PASs.

ANOVA showed that the storage-induced change in $\mathrm{pO}_{2}$ differed between the PASs $(P<0.05)$ with T-Sol PCs showing a continuous increase $(P<0.05)$ during storage while $\mathrm{pO}_{2}$ for the other PASs remained stable. $\mathrm{pCO}_{2}$ was significantly reduced by washing for all PASs followed by an increase during the storage period after washing. The storage-induced changes in $\mathrm{pCO}_{2}$ differed between PASs $(P<0.05)$.

Glucose and lactate concentrations were reduced to $<1 \mathrm{mmol} / \mathrm{l}$ by the washing procedure. The albumin concentration was reduced to $<0.3 \mathrm{~g} / \mathrm{l}$ (range $0.04-0.27 \mathrm{~g} / \mathrm{l}$ ) by washing.

\section{Platelet surface markers}

Spontaneous P-selectin expression (percentage of positive cells and/or mean fluorescence intensity, MFI) increased by washing and during storage for PLTs in all PASs but there was a difference in activation level between the PASs (Fig. 3 A, B). T-Sol PLTs were more activated than Composol PLTs, which were more activated than SSP+ PLTs before and after washing. ANOVA confirmed that the washing- and storage-induced change in P-selectin differed $(P<0.05)$ between the PASs.

The up-regulation of P-selectin MFI following TRAP-6 stimulation decreased by washing for PLTs in all PASs (Fig. 3 C) but differed between the PASs as confirmed by ANOVA $(P<0.05)$. T-Sol PLTs had lower response than PLTs in the other PASs $(P<0.05)$ and Composol PLTs had lower response than PLTs in SSP+ $(P<0.05)$ before and after washing. There was a significant decrease in the up-regulation of P-selectin during storage after washing for T-Sol PLTs whereas no significant change could be found for PLTs in Composol or in SSP+. 
The percentage of GPIb expressing PLTs remained at a high level (>96\%) after washing and during subsequent storage for PLTs in all PASs (data not shown). Washing did not affect GPIb, expressed as MFI, but storage after washing resulted in a reduction in GPIb on PLTs in T-Sol or in Composol whereas it was unchanged on SSP+ PLTs (Fig. 3 D). T-Sol PLTs had lower GPIb than PLTs in Composol or in SSP+ on all sampling occasions. SSP+ PLTs had higher GPIb than Composol PLTs from 1 day post wash. ANOVA confirmed that the effect of washing was similar $(P>0.05)$ for the different PASs and that changes in GPIb occurring during storage after washing differed $(P<0.05)$ between the PASs.

\section{Clotting time and coagulum elasticity ( $\left.G^{\prime}\right)$}

The clotting times and elasticity $\left(\mathrm{G}^{\prime}\right)$ values are shown in Table 2 . The clotting time decreased by washing for all PASs $(P<0.05)$ but T-Sol PCs had a larger decrease $(P<0.05)$ than PCs with the other PASs. Clotting time was not further altered during storage. ANOVA confirmed that the washing-induced change in clotting time differed $(P<0.05)$ between the PASs and that storage after washing affected the PASs to a similar extent $(P>0.05)$.

Washing did not affect the G' (G'max, time to G'max or mean change in G'/min) for PCs with any of the PASs which was confirmed by ANOVA $(P>0.05)$. Storage after washing did not affect time to G'max for PCs with any of the PASs but reduced G'max and mean change in G'/min significantly for T-Sol and Composol PCs while these variables remained unchanged for PCs with SSP+. 


\section{Discussion}

Washed PCs should be transfused shortly after washing according to guidelines [2].

Prolongation of the storage period of washed PLTs prior to transfusion would be logistically beneficial but requires that the PLT function is well maintained. Until now we have used TSol routinely for washing of PLTs but new solutions are now available for storage and washing. This study compares the quality of PCs washed with T-Sol, Composol or SSP+ on day 5 of storage and then stored for 2 more days (day 7) with the PAS. In our daily routine only swirling has been used to check the quality of washed PLTs, but in this study a number of different in vitro assays were used to assess the PLT quality. Overall, our findings support prolongation of the storage period for washed PCs prior to transfusion. PLTs washed with $\mathrm{SSP}+$ had better maintained in vitro quality compared to PLTs washed with T-Sol or Composol.

Presence of glucose during storage has been claimed to be necessary for maintenance of PLT function and viability [12]. T-Sol, Composol and SSP+ all contain acetate, which has been shown to reduce the need for glucose during storage. However, acetate alone has been claimed not to maintain full PLT function and energy levels during storage [13]. In contrast, Guppy et al. [14] showed that PLTs were better preserved in the absence of glucose and Whisson et al. [15] showed that PLTs can be stored in a PAS containing acetate but no glucose. Composol and SSP+ contain $\mathrm{KCl}$ and $\mathrm{MgCl}_{2}$, but SSP+ also contains phosphate and Composol gluconate $[3,16] . \mathrm{KCl}, \mathrm{MgCl}_{2}$ and phosphate have been shown to improve the storage conditions of PLTs [3, 17-20].

Washing reduced the PLT number to a similar degree for all the PASs (16-22\%) but less than previously reported $[5,8]$. T-Sol PCs, in contrast to PCs with Composol or SSP+, had a small 
but significant reduction in PLT number also during storage after washing. A loss of PLTs might lead to transfusion of more PCs to maintain a relevant number of circulating PLTs in the recipient [21].

Washing also resulted in a small but significant reduction in size (MPV) for PLTs in T-Sol or in SSP+, probably reflecting a more pronounced loss of larger cells. At the end of storage, MPV of T-Sol PLTs had increased significantly and to a higher level than for PLTs in the other PASs $(P<0.05)$. Such an increase in PLT size during storage has been suggested to be caused by change of the PLT shape from discoid to spherical [22].

We found $\mathrm{pH}$ to be acceptable [11] in PCs washed and stored with the different PASs. PCs with SSP+ had best preserved $\mathrm{pH}$ throughout storage, which might be due to the presence of phosphate [17].

GPIb is expressed on the surface of resting PLTs and becomes down-regulated following PLT activation $[23,24]$ whereas P-selectin is expressed only on activated PLTs [25, 26]. Washing and subsequent storage of PLTs with all PASs resulted in an increased PLT activation. The activation level of the washed PCs at the end of storage was comparable to what has been reported for unwashed PCs stored for 7 days [4, 27, 28]. However, it is difficult to compare Pselectin values from various studies due to differences in the analysis procedure. PLTs in TSol had higher spontaneous activation (lower GPIb and higher P-selectin expression) than PLTs in Composol or in SSP+. This is probably due to the presence of $\mathrm{KCl}$ and $\mathrm{MgCl}_{2}$ in Composol and SSP+ since these substances have been shown to reduce the PLT activation [3, 19, 20]. PLTs in Composol were spontaneously more activated than PLTs in SSP+. Ringwald et al. [5] also reported higher PLT activation for PLTs in Composol than in SSP+ and 
hypothesized that the more stable and higher $\mathrm{pH}$ of SSP+ PCs might have contributed to this. Washing reduced the PLT capacity to respond to the PLT agonist TRAP-6, most likely due to increased spontaneous activation. PLTs in SSP+ had best preserved capacity to respond to the agonist while T-Sol PLTs had a much lower response capacity than PLTs in Composol or in $\mathrm{SSP}+$. GPIb and P-selectin have been reported to be involved in the post transfusion clearance of PLTs $[29,30]$ and may affect the in vivo viability of PLTs [31-33] but other studies report only a weak or no correlation between activation level and in vivo viability $[34,35]$. The relevance of the differences in activation level found in this study therefore needs to be further investigated.

We assessed the haemostatic properties of the PLTs by FOR which shows the capacity of PLTs to participate in clot formation (clotting time) and clot retraction (elasticity). Washing shortened the clotting time, possibly reflecting the increased spontaneous PLT activation. TSol PCs had shortest clotting time directly after washing as well highest PLT activation. When PLTs become activated not only P-selectin is released from the PLT granules but also other pro-coagulant substances such as ADP, fibrinogen and coagulation factors [36] and such a release into the PC might account for the shortening of the clotting time. In contrast to our findings in this study, we did not find any effect of washing on the clotting time in a previous study when PLTs were washed with T-Sol on day 1 and 7 [8]. The ability of the PLTs to participate in clot retraction was not affected by washing with any of the PASs which is in accordance with our previous study [8]. However, by the end of storage after washing (2 days post wash) we found a small reduction in clot retraction for PLTs in T-Sol or in Composol $(P<0.05)$. 
By visual inspection we found that washing with Composol resulted in formation of microaggregates requiring a longer resting period after washing than for PCs with T-Sol or with $\mathrm{SSP}+$ to dissolve the aggregates. It is possible that the larger drop in $\mathrm{pH}$ after washing with Composol might have caused the formation of micro-aggregates. However, the formation of micro-aggregates did not result in an inferior quality of Composol PCs compared to T-Sol PCs as judged by the in vitro analyses we used.

In summary, we found that PLTs washed with T-Sol, Composol or SSP+ had good in vitro quality for up to 2 days after washing (well maintained swirling, $\mathrm{pH}$, and good ability to promote clot formation and clot retraction) despite the absence of glucose. However, when comparing the PASs, PCs washed with T-Sol showed less well preserved in vitro variables during storage after washing as seen by a reduction in PLT number, highest spontaneous PLT activation and lowest PLT response to an activation agonist while PLTs washed with SSP+ showed best in vitro quality. The results of this study support a prolongation of the storage period for washed PCs prior to transfusion and the use of SSP+ for PLT washing and storage.

\section{Acknowledgements}

The study was supported by the County Council of Östergötland and Linköping Medical Society. 


\section{References}

$1 \quad$ Stroncek DF, Rebulla P: Platelet transfusions. Lancet 2007; 370:427-438

2 Lockwood WB, Leonard J, Liles SL: Storage, monitoring, pretransfusion processing, and distribution of blood components; in Roback JD, Rae Combs M, Grossman BJ, Hillyer CD (ed): Technical Manual, 16th edition. Bethesda, American Association of Blood Banks, 2008

3 Gulliksson H, AuBuchon JP, Vesterinen M, et al.: Storage of platelets in additive solutions: a pilot in vitro study of the effects of potassium and magnesium. Vox Sang 2002; 82:131-136

4 Cardigan R, Sutherland J, Garwood M, et al.: In vitro function of buffy coat-derived platelet concentrates stored for 9 days in CompoSol, PASII or $100 \%$ plasma in three different storage bags. Vox Sang 2008; 94:103-112

5 Ringwald J, Althoff F, Zimmermann R, et al.: Washing platelets with new additive solutions: aspects on the in vitro quality after 48 hours of storage. Transfusion 2006; 46:236-243

6 Hirayama J, Azuma H, Fujihara M, et al.: Comparison between in vitro qualities of platelets washed with commercially available additive solutions and those washed with M-sol. Vox Sang 2010; 99:131-135

7 Tynngård N, Lindahl T, Ramström S, et al.: Effects of different blood components on clot retraction analysed by measuring elasticity with a free oscillating rheometer. Platelets 2006; 17:545-554

8 Tynngård N, Trinks M, Berlin G: Platelet quality after washing: the effect of storage time before washing. Transfusion 2010; 50:2745-2752 
9 Larsson S, Sandgren P, Sjodin A, et al.: Automated preparation of platelet concentrates from pooled buffy coats: in vitro studies and experiences with the OrbiSac system. Transfusion 2005; 45:743-751

10 Tynngård N, Lindahl TL, Trinks M, et al.: The quality of platelet concentrates produced by COBE Spectra and Trima Accel cell separators during storage for 7 days as assessed by in vitro methods. Transfusion $2008 ; \mathbf{4 8 : 7 1 5 - 7 2 2}$

11 Guide to the preparation, use and quality assurance of blood components, ed 13. Strasbourg Cedex, Council of Europe Publishing, 2007

12 Gulliksson H: Additive solutions for the storage of platelets for transfusion. Transfus Med 2000; 10:257-264

13 Holme S: Effect of additive solutions on platelet biochemistry. Blood Cells 1992; 18:421-430

14 Guppy M, Whisson ME, Sabaratnam R, et al.: Alternative fuels for platelet storage: a metabolic study. Vox Sang 1990; 59:146-152

15 Whisson ME, Nakhoul A, Howman P, et al.: Quantitative study of starving platelets in a minimal medium: maintenance by acetate or plasma but not by glucose. Transfus Med 1993; 3:103-113

16 van der Meer PF, Pietersz RN, Reesink HW: Comparison of two platelet additive solutions. Transfus Med 2001; 11:193-197

17 Shimizu T, Murphy S: Roles of acetate and phosphate in the successful storage of platelet concentrates prepared with an acetate-containing additive solution.

Transfusion 1993; 33:304-310

18 Gulliksson H, Larsson S, Kumlien G, et al.: Storage of platelets in additive solutions: effects of phosphate. Vox Sang 2000; 78:176-184 
19 de Wildt-Eggen J, Schrijver JG, Bins M, et al.: Storage of platelets in additive solutions: effects of magnesium and/or potassium. Transfusion 2002; 42:76-80

20 Ringwald J, Walz S, Zimmermann R, et al.: Hyperconcentrated platelets stored in additive solution: aspects on productivity and in vitro quality. Vox Sang 2005; 89:1118

21 Slichter SJ, Kaufman RM, Assmann SF, et al.: Dose of prophylactic platelet transfusions and prevention of hemorrhage. N Engl J Med 2010; 362:600-613

22 Sandgren P, Shanwell A, Gulliksson H: Storage of buffy coat-derived platelets in additive solutions: in vitro effects of storage at 4 degrees C. Transfusion 2006; 46:828834

23 Hourdille P, Heilmann E, Combrie R, et al.: Thrombin induces a rapid redistribution of glycoprotein Ib-IX complexes within the membrane systems of activated human platelets. Blood 1990; 76:1503-1513

24 Leytin V, Allen DJ, Gwozdz A, et al.: Role of platelet surface glycoprotein Ibalpha and P-selectin in the clearance of transfused platelet concentrates. Transfusion 2004;

44:1487-1495

25 Stenberg PE, McEver RP, Shuman MA, et al.: A platelet alpha-granule membrane protein (GMP-140) is expressed on the plasma membrane after activation. J Cell Biol $1985 ; 101: 880-886$

26 Kostelijk EH, Fijnheer R, Nieuwenhuis HK, et al.: Soluble P-selectin as parameter for platelet activation during storage. Thromb Haemost 1996; 76:1086-1089

27 Hornsey VS, McColl K, Drummond O, et al.: Extended storage of platelets in SSP+ platelet additive solution. Vox Sang 2006; 91:41-46

28 Zhang JG, Carter CJ, Culibrk B, et al.: Buffy-coat platelet variables and metabolism during storage in additive solutions or plasma. Transfusion 2008; 48:847-856 
29 Rinder HM, Bonan JL, Rinder CS, et al.: Dynamics of leukocyte-platelet adhesion in whole blood. Blood 1991; 78:1730-1737

30 Hoffmeister KM, Felbinger TW, Falet H, et al.: The clearance mechanism of chilled blood platelets. Cell 2003; 112:87-97

31 Rinder HM, Murphy M, Mitchell JG, et al.: Progressive platelet activation with storage: evidence for shortened survival of activated platelets after transfusion. Transfusion 1991; 31:409-414

32 Sweeney JD, Holme S, Moroff G: Storage of apheresis platelets after gamma radiation. Transfusion 1994; 34:779-783

33 Dumont LJ, AuBuchon JP, Whitley P, et al.: Seven-day storage of single-donor platelets: recovery and survival in an autologous transfusion study. Transfusion 2002; 42:847-854

34 Holme S, Sweeney JD, Sawyer S, et al.: The expression of p-selectin during collection, processing, and storage of platelet concentrates: relationship to loss of in vivo viability. Transfusion 1997; 37:12-17

35 Slichter SJ, Bolgiano D, Jones MK, et al.: Viability and function of 8-day-stored apheresis platelets. Transfusion 2006; 46:1763-1769

36 Jurk K, Kehrel BE: Platelets: physiology and biochemistry. Semin Thromb Hemost $2005 ; 31: 381-392$ 
Fig.1. A typical FOR curve showing the changes in clot elasticity $\left(\mathrm{G}^{\prime}, \mathrm{Pa}\right)$ over time in a PC with T-Sol with clotting time, maximum elasticity (G'max) and time to G'max indicated.

Fig.2. PLT number (A) and mean PLT volume (B) for PLTs in T-Sol, Composol or SSP+ pre wash, post wash, 1 day post wash and 2 days post wash.

* Significant difference $(P<0.05)$ compared to day 5 after washing within each PAS group. Results are expressed as mean $+\mathrm{SD}$ for $n=10$.

Fig.3. Flow cytometry data for PLTs in T-Sol, Composol or SSP+ pre wash, post wash, 1 day post wash and 2 days post wash. Spontaneous P-selectin expression as percentage of positive PLTs (A) and as mean fluorescence intensity (MFI;B) in arbitrary units (AU). The upregulation of P-selectin MFI following TRAP-6 stimulation (C). Spontaneous expression of GPIb measured as MFI (D).

* Significant difference $(P<0.05)$ compared to day 5 after washing within each PAS group. Results are expressed as mean + SD for $n=10$. 
Table 1. Blood gas variables of PCs with T-Sol, Composol or SSP+

Results are expressed as mean $\pm \mathrm{SD}(n=10)$. Statistical differences between storage days are indicated separately for PCs in T-Sol, Composol or SSP+.

${ }^{\mathrm{a}}=P<0.05$ vs. post wash.

${ }^{\mathrm{b}}=P<0.05$ vs. $\mathrm{T}-$ Sol, ${ }^{\mathrm{c}}=P<0.05$ vs. Composol.

Table 2. FOR variables of PCs with T-Sol, Composol or SSP+

Results are expressed as mean $\pm \mathrm{SD}(n=10)$. Statistical differences between storage days are indicated separately for PCs in T-Sol, Composol or SSP+.

${ }^{\mathrm{a}}=P<0.05$ vs. post wash.

${ }^{\mathrm{b}}=P<0.05$ vs. $\mathrm{T}-\mathrm{Sol},{ }^{\mathrm{c}}=P<0.05$ vs. Composol. 


\section{Table 1}

$\begin{array}{llllll}\text { Variable } & \text { PC type } & \text { Pre wash } & \text { Post wash } & \text { 1 day post wash } & \text { 2 days post wash } \\ \text { pH } & \text { T-Sol } & 7.13 \pm 0.05^{\mathrm{ac}} & 6.90 \pm 0.03 & 6.81 \pm 0.02^{\mathrm{a}} & 6.90 \pm 0.04 \\ \left(37^{\circ} \mathrm{C}\right) & \text { Composol } & 7.20 \pm 0.05^{\mathrm{ab}} & 6.88 \pm 0.04 & 6.80 \pm 0.05^{\mathrm{a}} & 6.90 \pm 0.05 \\ & \text { SSP+ } & 7.13 \pm 0.04^{\mathrm{c}} & 7.16 \pm 0.01^{\mathrm{bc}} & 7.12 \pm 0.01^{\mathrm{abc}} & 7.11 \pm 0.01^{\mathrm{abc}} \\ \mathbf{p O}_{2} & \text { T-Sol } & 17.6 \pm 1.2 & 18.1 \pm 1.4 & 18.7 \pm 1.8 & 20.2 \pm 1.4^{\mathrm{a}} \\ (\mathrm{kPa}) & \text { Composol } & 18.0 \pm 1.4^{\mathrm{a}} & 18.9 \pm 1.7 & 18.3 \pm 1.3^{\mathrm{a}} & 18.7 \pm 2.0 \\ & \text { SSP+ } & 17.8 \pm 1.6 & 18.4 \pm 1.0 & 17.9 \pm 1.3 & 18.6 \pm 2.2 \\ \mathbf{p C O} & \text { T-Sol } & 2.5 \pm 0.2^{\mathrm{a}} & 0.6 \pm 0.1 & 1.0 \pm 0.1^{\mathrm{a}} & 1.0 \pm 0.1^{\mathrm{ac}} \\ (\mathrm{kPa}) & \text { Composol } & 2.3 \pm 0.2^{\mathrm{a}} & 0.5 \pm 0.1 & 1.1 \pm 0.1^{\mathrm{a}} & 1.2 \pm 0.1^{\mathrm{ab}} \\ & \text { SSP+ } & 2.1 \pm 0.2^{\mathrm{ab}} & 0.3 \pm 0.0^{\mathrm{bc}} & 0.7 \pm 0.1^{\mathrm{abc}} & 1.1 \pm 0.1^{\mathrm{ab}}\end{array}$


Table 2

Variable PC type Pre wash Post wash 1 day post wash 2 days post wash

\section{Clotting time}

\begin{tabular}{|c|c|c|c|c|c|}
\hline \multirow[t]{2}{*}{$(\min )$} & T-Sol & $10.7 \pm 1.9^{\mathrm{a}}$ & $8.2 \pm 1.4^{\mathrm{c}}$ & $9.3 \pm 1.4$ & $8.8 \pm 1.9$ \\
\hline & Composol & $10.2 \pm 1.3^{\mathrm{a}}$ & $9.1 \pm 1.9^{b}$ & $9.0 \pm 1.8$ & $8.7 \pm 2.0$ \\
\hline & $\mathrm{SSP}+$ & $10.7 \pm 1.7^{\mathrm{a}}$ & $9.2 \pm 1.3^{b}$ & $9.4 \pm 1.8$ & $9.0 \pm 2.3$ \\
\hline
\end{tabular}

\section{G'max}

$(\mathrm{Pa})$

$\begin{array}{lcccc}\text { T-Sol } & 1339 \pm 122 & 1350 \pm 197 & 1250 \pm 90^{\mathrm{a}} & 1167 \pm 120^{\mathrm{a}} \\ \text { Composol } & 1365 \pm 73 & 1370 \pm 99 & 1296 \pm 98^{\mathrm{a}} & 1201 \pm 196^{\mathrm{a}} \\ \text { SSP+ } & 1360 \pm 98 & 1373 \pm 71 & 1375 \pm 224 & 1328 \pm 125^{\mathrm{b}}\end{array}$

Time to G'max

\begin{tabular}{|c|c|c|c|c|}
\hline T-Sol & $35.2 \pm 1.9$ & $33.7 \pm 2.3^{c}$ & $34.1 \pm 2.2$ & $34.6 \pm 1.5$ \\
\hline Composol & $35.4 \pm 1.8$ & $35.3 \pm 1.7^{\mathrm{b}}$ & $35.4 \pm 2.0$ & $34.6 \pm 2.0$ \\
\hline $\mathrm{SSP}+$ & $37.0 \pm 2.0^{\mathrm{b}}$ & $37.4 \pm 3.5^{b}$ & $37.4+2.1^{b}$ & $36.7 \pm 1$ \\
\hline
\end{tabular}

Mean change in $\mathbf{G}^{\prime} / \mathrm{min}$

$\begin{array}{llllll}(\mathrm{Pa} / \mathrm{min}) & \text { T-Sol } & 38 \pm 3 & 40 \pm 5 & 37 \pm 3 & 34 \pm 4^{\mathrm{a}} \\ & \text { Composol } & 39 \pm 3 & 39 \pm 3 & 37 \pm 3 & 35 \pm 7^{\mathrm{a}} \\ & \mathrm{SSP}+ & 37 \pm 4 & 37 \pm 4 & 37 \pm 6 & 37 \pm 4\end{array}$




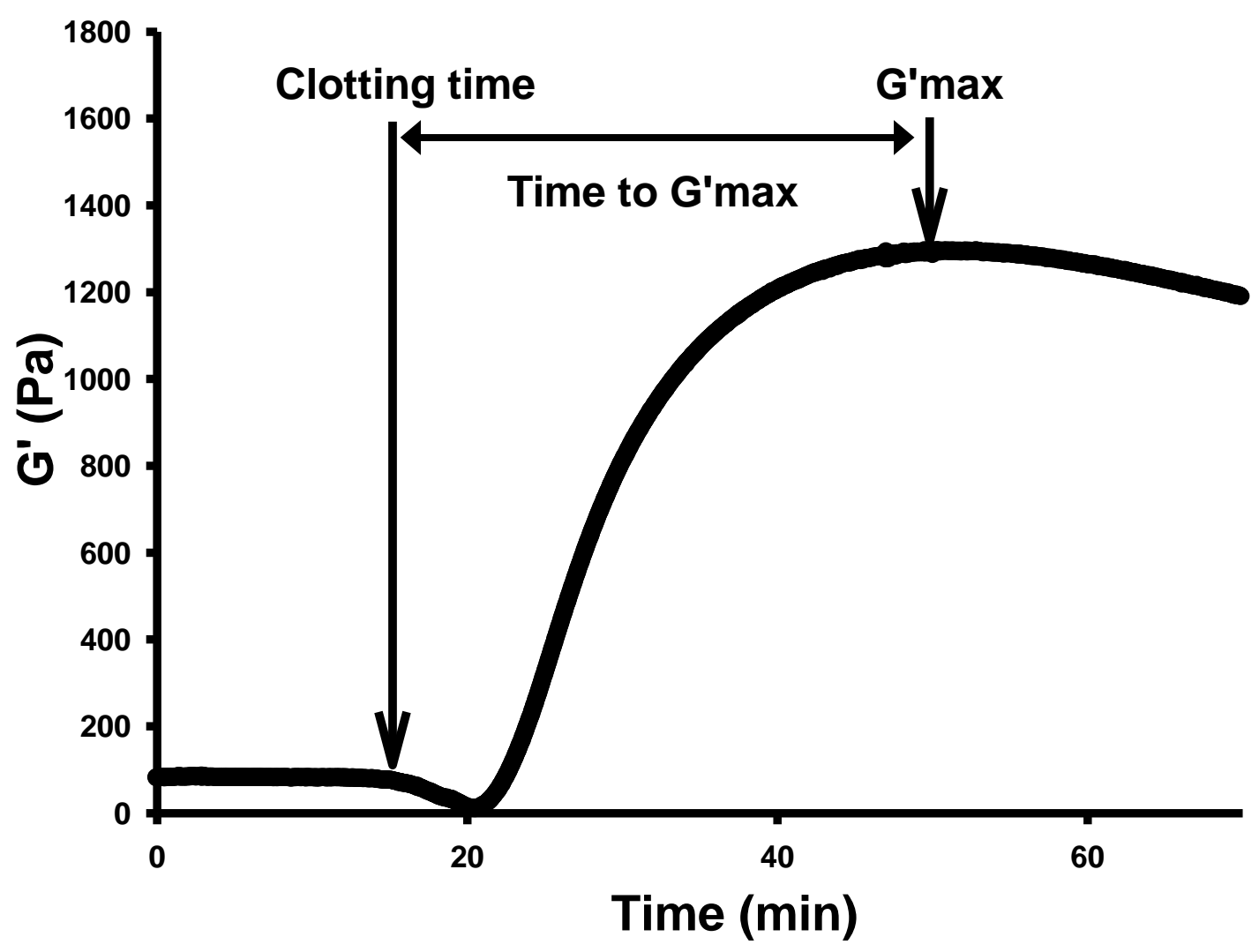

Fig.1 

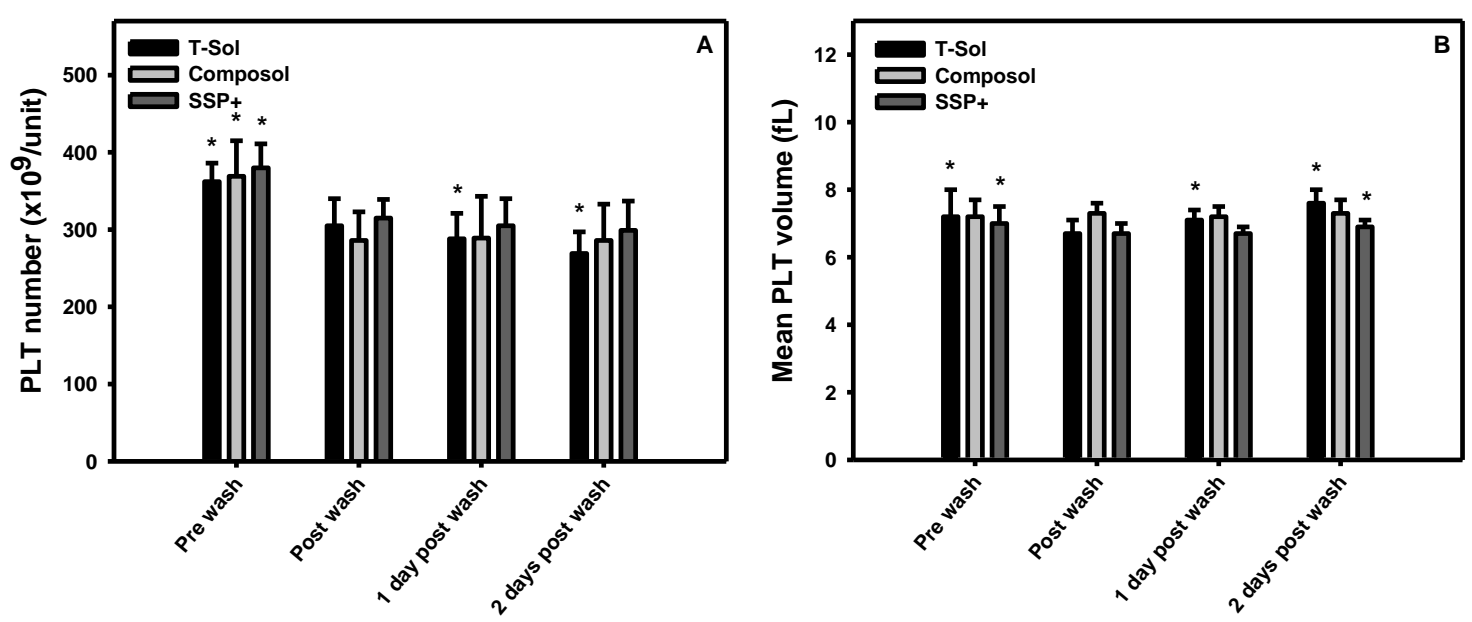

Fig.2A-B 

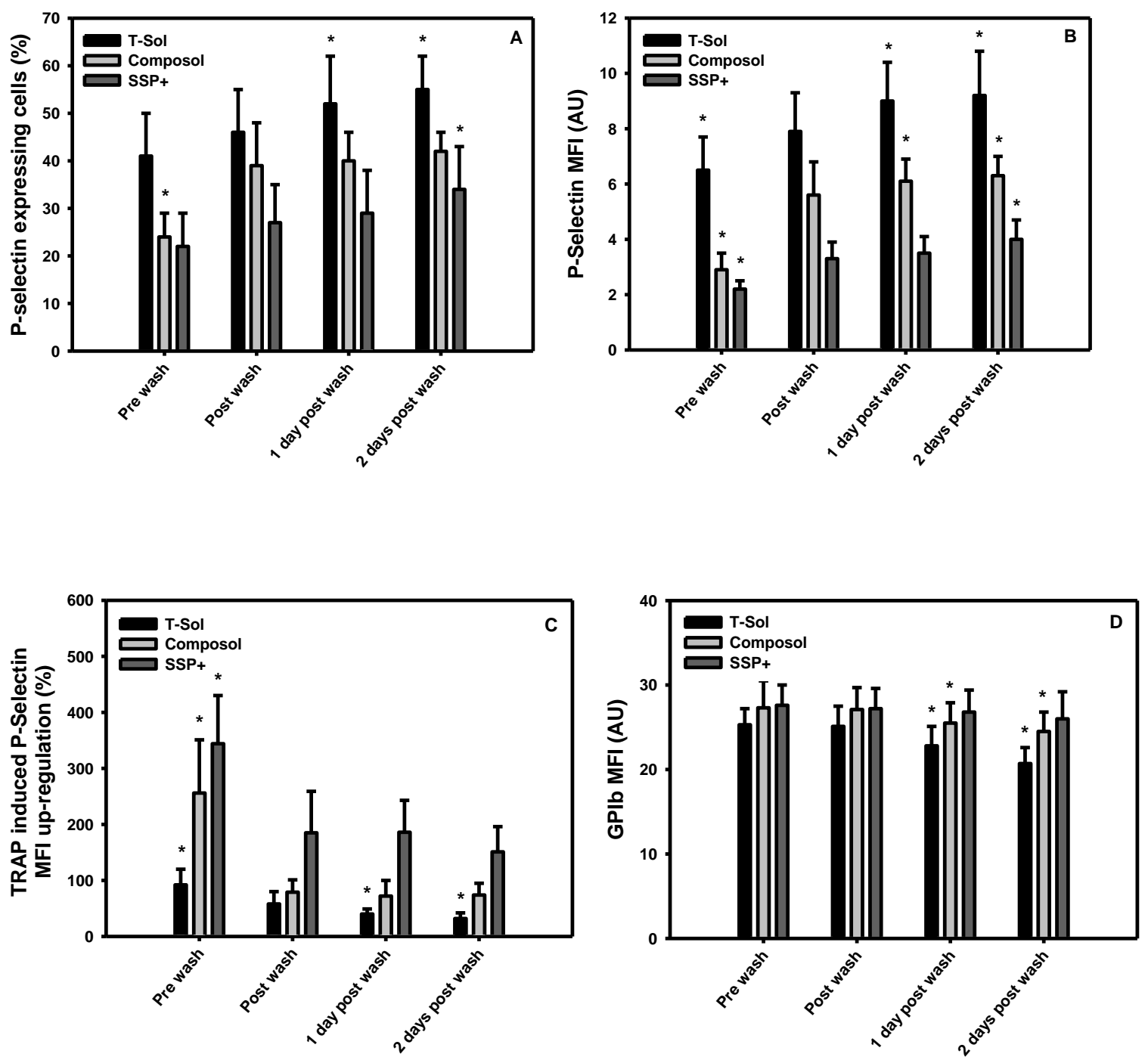

Fig. 3A-D 